\title{
Perfil clínico-epidemiológico de pacientes diagnosticados com câncer gástrico atendidos na especialidade de oncologia do Hospital de Clínicas de Passo Fundo - RS
}

Julia Pastorello1, Emanuela Lando ${ }^{*}$, André Lunardi Mondadori Messaggi², Marina Ractz Bueno', Cristiane Pagnussat Cechetti ${ }^{1}$, Camila dos Santos do Amaral' , Douglas Carvalho Cogo', Luiz Artur Rosa Filho $^{3}$

${ }^{1}$ Departamento de Oncologia Clínica, Hospital de Clínicas de Passo Fundo - HCPF, Passo Fundo, Rio Grande do Sul/RS, Brasil.

${ }^{2}$ Acadêmicos de Medicina. Faculdade Meridional - IMED, Passo Fundo, Rio Grande do Sul/RS, Brasil.

${ }^{3}$ Professor do curso de Medicina, Faculdade Meridional - IMED, Passo Fundo, Rio Grande do Sul/RS, Brasil.

*Autor Correspondente: Emanuela Lando. Rua Paisandú,358. Cep: 99010100 - Passo Fundo, Rio Grande do Sul/RS. Brasil. Fone: +55 (59) 9 91861467. E-mail: manu.lando@hotmail.com

Research Ethics Committee Approval (if necessary): Aprovado pelo Comitê de Ética da Faculdade Meridional - IMED (3.545.773).

Received on: Jul 8, 2021. Accepted on: Jul 21, 2021. Available online: Jul 23, 2021.

\section{Resumo}

O câncer gástrico é uma das neoplasias mais frequentes do mundo, ocupando o quarto lugar em frequência e o segundo em mortalidade. A origem do câncer gástrico é considerada multifatorial. Este estudo objetiva caracterizar o perfil clínicoepidemiológico dos pacientes com diagnóstico de câncer gástrico atendidos no Hospital de Clínicas de Passo Fundo no período de 2007 a 2016, realizado através de um estudo descritivo, transversal, quantitativo de caráter retrospectivo. No total foram 501 registros de pacientes com câncer gástrico analisados, sendo que a analise confirma os dados do perfil estudado dos pacientes envolvidos.

Keywords: Câncer Gástrico; Epidemiologia; Oncologia.

\section{Introdução}

O câncer gástrico (CG) é descrito na literatura há milhares de anos e no século XVIII, foi considerado como o câncer mais comum e mortal. Constitui a primeira neoplasia maligna do trato digestivo, sendo o quarto em frequência e o segundo em mortalidade. Apesar dos avanços no seu diagnóstico, a doença geralmente é detectada após a invasão da camada muscular própria [1].

No ano de 2018, no mundo, os novos casos de câncer gástrico se encontraram entre quarta e quinta posições, para o sexo masculino e feminino respectivamente, entre os tipos 
de cânceres mais prevalentes. A maior incidência por idade é entre 50 e 70 anos, com uma incidência máxima em torno dos 60 anos, sendo pouco frequente antes dos 30 anos [2].

As regiões brasileiras com maior concentração de tumor gástrico são a Sudeste e Sul e segundo o INCA, no ano de 2018 a estimativa de novos casos foi de 21.290 [3].

No entanto, apesar do declínio da incidência dessa malignidade, na população mundial, tem sido verificado um aumento de casos em pacientes jovens [3-4].

Considera-se multifatorial a origem do câncer gástrico e tem variação conforme a localização geográfica, etnia, fatores genéticos, ambientas, hábitos de vida e ou exposição a agentes oncogênicos [5 -7].

O presente trabalho visa em essência caracterizar o perfil clínico e epidemiológico de pacientes diagnosticados com câncer gástrico atendidos no Hospital de Clínicas de Passo Fundo - HCPF.

\section{Metodologia}

Trata-se de um estudo descritivo, transversal, quantitativo e de caráter retrospectivo realizado no HCPF. A população estudada compreende todos os pacientes com câncer gástrico atendidos no HCPF, no período de 2007 a 2016.

A identificação das neoplasias foi realizada na base de dados do registro hospitalar de câncer. Para descrever o comportamento das variáveis de exposição em relação ao desfecho foram analisadas de maneira categórica usando o Software SPSS. As variáveis analisadas foram: sexo, idade de diagnóstico, escolaridade, etnia, estado civil, tabagismo, etilismo, histórico familiar, sublocalização anatômica, primeiro tratamento recebido no hospital, estadiamento e óbitos.

Foram incluídos todos os pacientes com câncer gástrico atendidos no HCPF, no período de 2007 a 2016. Não houve exclusão de pacientes.

\section{Resultados}

No estudo, foram analisados todos os 501 prontuários presentes no registro hospitalar de câncer de pacientes diagnosticados com câncer gástrico. Aproximadamente $67 \%$ são do sexo masculino e $33 \%$ do sexo feminino. Quanto a idade de diagnóstico, 26 (5\%) dos pacientes foram diagnosticados com menos de 40 anos, 51 (10\%) entre 40 e 49 anos, 115 (23\%) 50 e 59 anos, 127 (25\%) 60 e $69,13(27 \%) 70$ e 79 anos e $49(10 \%)$ com mais de 80 anos. A maior parte dos casos estão concentrados entre os 50 e 79 anos (75\%), com maior incidência entre os $70-79$ anos (27\%) (Tabela 1).

Observou-se a predominância de estado civil casado em 340 (68\%), seguido por solteiro $66(13 \%)$, viúvo 59 $(12 \%)$, separado $25 \quad(5 \%)$ e união consensual $6(1 \%)$. Constava como sem informação 5 (1\%) dos pacientes.

No que diz respeito à etnia, a branca apresentou maior amostra com 463 (92\%), seguido da parda 17 (3\%) e preta $7(1 \%)$. A maior parte da população observada era natural do estado do Rio Grande do Sul (n=451, $90 \%)$. 
A respeito do nível de escolaridade, $32(6 \%)$ dos pacientes apresentavam nenhum nível, 275 (55\%) fundamental incompleto, 56 (11\%) fundamental completo, 36 (7\%) nível médio, $3(1 \%)$ superior incompleto, 28 (6\%) superior completo e 71 (14\%) não havia informação (Tabela 1$)$.

O tabagismo é um importante fator de risco para o desenvolvimento do câncer gástrico. Na presente análise, $146(29 \%)$ eram tabagistas, 69 (14\%) exconsumidores, 145 (29\%) nunca haviam fumado e 141 (28\%) sem informação. Já em relação ao etilismo, 69 (14\%) consumiam, 61 (14\%) eram exconsumidores, 211 (42\%) nunca haviam consumido e 160 (32\%) sem informação. A história familiar positiva da neoplasia estava presente em 115 (23\%) e não apresentava história familiar em 146 (29\%) dos pacientes. O histórico familiar não tinha informação em 240 (48\%) dos registros hospitalares (Tabela 2).

A base mais importante do diagnóstico foi a histologia do tumor primário $(80 \%)$. Em relação às características do tumor, observou-se que as localizações primárias mais acometidas foram o corpo 119 (23,8\%), seguido do antro $102(20,4 \%)$ e cardia 52 $(10,4 \%)$. Quanto a localização, a maioria eram na região proximal 178 (35\%). O tipo histológico mais comum foi $\mathrm{o}$ adenocarcinoma 268 (53,8 \%).

Em relação ao primeiro tratamento recebido no hospital para o câncer gástrico, grande parte dos pacientes $200 \quad(39,9 \%)$ realizaram a quimioterapia, seguido de terapia combinada de cirurgia e quimioterapia $116(23,2 \%)$ e somente cirurgia em 64 $(12,8 \%)$. Além disso, não houve tratamento recebido no hospital em 117 $(23,4 \%)$ dos pacientes. Quanto ao estadiamento clínico, a maioria dos pacientes 162 (32,3\%) apresentavam metástases a distância, estádio 4, seguido do estádio 3 com 72 (14,4\%), estádio 2 com 49 (9.8\%) e estádio 1 com $30(6 \%)$. No estadiamento não havia informação em $136(27,1 \%)$ e constava como não se aplica em $52(10,4 \%)$ dos registros (Tabela 3 ).

No presente estudo, houveram 191 óbitos. Destes, 131 óbitos eram confirmados pelo câncer gástrico.

Table 1. Distribuição dos pacientes com CG, segundo as variáveis idade de diagnóstico, gênero e nível de escolaridade.

\begin{tabular}{|c|c|}
\hline Variáveis & $\mathrm{n}=501(100 \%)$ \\
\hline \multicolumn{2}{|l|}{ Idade Diagnóstico (anos) } \\
\hline$<40^{\circ}$ & $26(5 \%)$ \\
\hline $40-49$ & $51(10 \%)$ \\
\hline $50-59$ & $115(23 \%)$ \\
\hline $60-69$ & $127(25 \%)$ \\
\hline 70-79 & $133(27 \%)$ \\
\hline $80+$ & $49(10 \%)$ \\
\hline \multicolumn{2}{|l|}{ Gênero } \\
\hline Masculino & $335(67 \%)$ \\
\hline Feminino & $166(33 \%)$ \\
\hline Nível Escolaridade & \\
\hline Nenhuma & $32(6 \%)$ \\
\hline
\end{tabular}


Perfil clínico-epidemiológico de pacientes diagnosticados com câncer gástrico

Fundamental incompleto

$275(55 \%)$

Fundamental completo

$56(11 \%)$

Nível médio

$36(7 \%)$

Superior completo

$28(6 \%)$

Tabela 2. Distribuição dos pacientes com CG, segundo as variáveis de tabagismo, alcoolismo e histórico familiar de câncer gástrico.

\begin{tabular}{|c|c|}
\hline Variáveis & $\mathrm{n}=501(100 \%)$ \\
\hline \multicolumn{2}{|l|}{ Tabagismo } \\
\hline Nunca & $145(29 \%)$ \\
\hline Ex-consumidor & $69(14 \%)$ \\
\hline Sim & $146(29 \%)$ \\
\hline Sem informação & $141(28 \%)$ \\
\hline \multicolumn{2}{|l|}{ Alcoolismo } \\
\hline Nunca & $211(42 \%)$ \\
\hline Ex-consumidor & $61(12 \%)$ \\
\hline Sim & $69(14 \%)$ \\
\hline Sem informação & $160(32 \%)$ \\
\hline \multicolumn{2}{|l|}{ Histórico Familiar } \\
\hline Sim & $115(23 \%)$ \\
\hline Não & $146(29 \%)$ \\
\hline Sem informação & $240(48 \%)$ \\
\hline
\end{tabular}

Tabela 3. Distribuição dos pacientes com CG, segundo as variáveis de localização primária do tumor e estadiamento TNM.

\begin{tabular}{|c|c|}
\hline Variáveis & $\mathrm{n}=501(100 \%)$ \\
\hline \multicolumn{2}{|l|}{ Localização } \\
\hline Cardia & $52(10,4 \%)$ \\
\hline Fundo & $7(1,4 \%)$ \\
\hline Corpo & $119(23,8 \%)$ \\
\hline Antro & $102(20,4 \%)$ \\
\hline Piloro & $8(1,6 \%)$ \\
\hline Invasivo & $5(1 \%)$ \\
\hline Não informado & $208(41,5 \%)$ \\
\hline \multicolumn{2}{|l|}{ Estadiamento } \\
\hline I & $30(6 \%)$ \\
\hline II & $49(9,8 \%)$ \\
\hline III & $72(14,3 \%)$ \\
\hline IV & $162(32,3 \%)$ \\
\hline Não se aplica & $52(10,4 \%)$ \\
\hline Sem informação & $136(27,1 \%)$ \\
\hline
\end{tabular}

\section{Discussão e Conclusão}

Conforme a análise do registro hospitalar de câncer observou-se que a maioria dos pacientes com câncer gástrico são do sexo masculino. Este resultado confirma os estudos internacionais onde as taxas são mais elevadas no sexo masculino tanto em países Asiáticos como também na 
América do Sul. No continente Sul Americano, a incidência do sexo masculino é de $12.7 / 100.000$ e de 6.9/100.000 pessoas para o sexo feminino [8].

Estudos nacionais também demonstraram um maior acometimento do sexo masculino em relação ao feminino, respectivamente $63,3 \%$ e 59,5\%. [9 - 10] Questões como a ingesta de menor quantidade de frutas e hortaliças, maior consumo de refrescos artificias, refrigerantes, carnes, sal e alimentos processados pelos homens podem estar relacionados a um maior acometimento do sexo masculino como demonstra estudo prévio [11].

No que se refere a naturalidade e etnia, a maioria era natural do estado do Rio Grande do Sul (90\%) e 92\% tinham a etnia branca. Segundo IBGE, no estado do Rio Grande do Sul, a população autodeclara-se branca em $82,3 \%$, pardos $11,4 \%$, pretos $5,9 \%$ e amarelos ou indígenas $0,4 \%$ [12].

A incidência do câncer gástrico é maior em indivíduos com idade mais avançada. Crew e Neugut [13] relataram que a incidência aumenta conforme o avanço da idade e o pico observa-se entre 50 e 70 anos [13]. Este estudo corrobora com essa análise, pois a maior parte dos casos está entre os 50 a 79 anos.

Segundo Basaran et al. [14], menos de $10 \%$ de todos os pacientes tem uma faixa etária menor que 40 anos [14]. No presente estudo, somente $5 \%$ dos pacientes apresentaram idade menor que 40 anos. Sabe-se que, com o envelhecimento, os efeitos deletérios de fatores como risco ambiental e estilo de vida não favoráveis acumulam-se no organismo e alteram os mecanismos de proteção e reparação da mucosa gástrica e levam ao desenvolvimento de neoplasias [2-4].

$O$ câncer gástrico tem uma maior incidência em indivíduos com baixo nível socioeconômico. Estudos baseados em censos educacionais, renda familiar e ocupação mostrou a associação do câncer gástrico e o baixo nível socioeconômico, onde a taxa de indivíduos com menor nível socioeconômico pode ser de 2-3 vezes maior para o desenvolvimento da neoplasia em relação a classes mais altas, de tal modo que Nomura considera o câncer gástrico como uma doença de indivíduos com menores condições econômicas [15].

Uma das possibilidades para este achado é que indivíduos com baixa instrução educacional, geralmente, desconhecem de uma boa nutrição como baixa ingesta de sal, frituras e embutidos. Somado a isso, a deficiência do saneamento básico leva a uma somatização com a infecção pela bactéria Helicobacter pylori. Desde 1959, o consumo de sal é descrito como um possível fator de risco para câncer gástrico. Os mecanismos envolvidos incluem a potencialização da colonização e virulência de $\mathrm{H}$. pylori; alteração da camada muco protetora, principalmente pela exposição a compostos carcinogênicos como Nnitroso; e inflamação do epitélio gástrico, o que leva ao aumento da proliferação celular epitelial sendo esta parte do processo e probabilidade de mutação endógena [16]. 
A baixa escolaridade dos pacientes com câncer gástrico pode ser associado alimentação de pouca qualidade, menos acesso ao saneamento básico e a serviços de saúde. Neste estudo, não foram analisados dados de renda familiar ou ocupação, porém explorou-se o nível de escolaridade onde ficou evidente a elevada quantidade de indivíduos com câncer gástrico e baixo nível educacional, onde $72 \%$ das pessoas haviam estudado, somente, até o ensino fundamental completo.

O risco de câncer gástrico está relacionado ao hábito de fumar [5-7]. Diversos estudos demonstram evidências na relação quanto ao hábito de fumar e o câncer gástrico. Segundo estudo de González, qual demonstrou através de um estudo tipo metanálise, que aproximadamente $18 \%$ dos casos de câncer gástrico estão relacionados ao hábito de fumar [17]. Outro estudo fez a associação entre o risco de fumar e câncer gástrico: fumar 30 cigarros ao dia tem um risco de desenvolver câncer gástrico de 1,62 em homens e 1,2 em mulheres [18]. No atual estudo, 29\% eram tabagistas e $14 \%$ ex-tabagistas, ou seja, $43 \%$ dos pacientes analisados fizeram o uso do tabaco.

Segundo dados de um estudo, em 2018, realizado nas 26 capitais brasileiras e no Distrito Federal com adultos maiores de 18 anos, o percentual total de fumantes no Brasil é de 9,3\%, sendo $12,1 \%$ entre homens e $6,9 \%$ entre mulheres [19]. Neste estudo, observa-se um elevado número de pacientes que consumiam cigarro, o que indica uma provável relação do câncer gástrico com o tabagismo.

Também, observa-se que há um maior consumo de cigarro por homens em relação as mulheres em nível nacional [19]. Nesta análise, o câncer gástrico mostrou-se maior predisposição em indivíduos do sexo masculino o que leva a crer a uma tendência em associar câncer gástrico com tabagismo e sexo masculino.

Por outro lado, a maioria dos indivíduos nunca haviam consumido álcool. Conforme o Levantamento Nacional de Álcool e Drogas (LENAD), $32 \%$ da população brasileira ingere álcool de forma moderada e $16 \%$ consomem de forma nociva [20]. No estudo realizado, $12 \%$ eram exconsumidores e $14 \%$ consumidores, valor abaixo ao hábito de consumo brasileiro.

Em relação ao histórico familiar, quase a metade dos registros não apresentavam informação, o que prejudicou a análise, mas dentre aqueles com câncer gástrico $23 \%$ tinham história familiar o que é um dado bastante relevante. Estudos em relação ao consumo de álcool e o desenvolvimento de câncer gástrico ainda são controversos. Bartard et al. [21] e Tramacere et al. [22], em suas análises, não demonstraram associação entre $\mathrm{o}$ consumo de álcool e o desenvolvimento de CG. Além do mais, o consumo diário de vinho em baixas doses pode ser um fator protetor [21-22]. No entanto, outros estudos epidemiológicos sugerem que o álcool possui papel fundamental na carcinogênese, ao causar lesões na mucosa, principalmente 
quando age sinergicamente com o tabaco [21-23].

Acredita-se que o álcool atue de forma indireta promovendo o aumento de acidez e reduzindo propriedades do suco gástrico que promovem proteção a mucosa [23].

Embora o presente estudo não tenha demonstrado elevada quantidade de pacientes que eram etilistas, não se pode desconsiderar $\mathrm{o}$ fato de que o álcool é nocivo ao organismo humano e de que provavelmente tenha relação sinérgica a outros maus hábitos, como tabagismo, obesidade e dieta alimentar pobre em verduras e hortaliças.

Indivíduos com história familiar apresentam elevada incidência de Câncer Gástrico. Brenner et al, realizou um estudo de base populacional, casocontrole na Alemanha para avaliar as atribuições individuais e conjuntas de história familiar e infecção por H. pylori para o risco de carcinoma gástrico. Verificou-se que, embora a infecção por H. pylori e a história familiar estivessem relacionadas, ambos estavam independentemente e fortemente associados com o risco de câncer gástrico [24]. Neste estudo, o histórico familiar esteve presente em $23 \%$ dos pacientes. No entanto, houve um elevado número de registros sem informação (48\%), o que torna a análise de histórico familiar pouco relevante pela falta de dados.

No tocante a localização do tumor primário, observa-se diferenças conforme diferentes regiões geográficas, etnias e condições socioeconômicas. No estudo de Csendes e colaboradores, qual demonstram que a incidência do câncer no antro e corpo tem diminuído significativamente de forma global. Entre as razões para a essa diminuição podem estar relacionadas com o aumento da refrigeração e da disponibilidade de frutas e verduras frescas, com uma redução de 30\% nos casos da doença; diminuição de comida salgada e enlatados; aumento de consumo de produtos lácteos; diagnóstico de casos de câncer com antecedência; redução da infecção por Helicobacter pylori por melhor condição sanitária e tratamento [18].

No presente estudo, a maioria dos tumores estavam localizados no corpo $(23,8 \%)$, seguida do antro $(20,4 \%)$. Este dado pode revelar que, pelo fato do Brasil não ser um país considerado desenvolvido, a população pode ainda encontrar dificuldade em diagnóstico precoce, condições sanitárias e alimentação saudável. No entanto, houve um considerável número de localizações não informadas $(41,5 \%)$ o que prejudica sobremaneira a análise desta variável.

Em nível global, observou-se aumento do câncer na cardia. Esse aumento foi relacionado com comorbidades como refluxo gastroesofágico crônico e também com o aumento da obesidade global. Sugere-se que o carcinoma na cardia pode ter uma diferente etiologia em comparação com o restante dos carcinomas gástricos [25]. No atual estudo, 10,4\% dos pacientes tiveram a cardia como a localização primária do tumor.

No Brasil, segundo IBGE, um em cada cinco brasileiros são obesos. Também, o Banco de dados do 
Observatório Global da Organização Mundial da Saúde, demonstrou que a América Latina apresenta taxas semelhantes de obesidade como as regiões Asiática, Europeia e Oceania [12]. Portanto, o tumor localizado na cardia pode estar associada com a elevada massa corporal, pois a população local apresenta considerável relação com a obesidade.

Em relação ao estadiamento, o CG é descoberto em sua grande maioria já em estágios avançados (III ou IV), com sobrevida de $33,4 \%$ e $2 \%$, respectivamente [7]. Neste estudo, a maioria dos pacientes eram estádio 4 $(32,3 \%)$, seguido do estágio $3(14,3 \%)$. Portanto, é evidente que o câncer gástrico é diagnosticado tardiamente, isso pode ser explicado pela precariedade de disponibilidade de métodos mais tecnológicos para diagnóstico e rastreamento deste tipo de neoplasia no Brasil em comparação com países, como Japão, onde o diagnóstico é realizado de forma mais precoce. Assim, devido a esses fatores, o CG apresentase com elevada mortalidade.

Na presente análise, fica evidente que o número de óbitos é mais elevado que o descrito no registro hospitalar, pois estes dados são de pacientes que vem ao óbito no próprio hospital e os indivíduos que vem a falecer fora da instituição, geralmente, não são contabilizados. No atual estudo, houveram 191 (38\%) óbitos, sendo que 131 (26\%) eram confirmados pelo câncer gástrico.

O resultado da análise do perfil clínico epidemiológico de câncer gástrico é compatível com a literatura, sendo mais incidente no sexo masculino, em pacientes acima de 50 anos e na população com baixa instrução educacional [2-4]. $\mathrm{O}$ aumento da população idosa pode manter a ocorrência da doença em níveis crescentes. O hábito de fumar mostrouse elevado em cerca de 4 vezes a prevalência da população, no entanto, $o$ mesmo não se pode afirmar para o consumo de álcool.

Quanto ao histórico familiar houve um grande número de registros sem informação, fato que pode ser aprimorado no atendimento. As localizações dos tumores foram mais incidentes na região do corpo e antro.

As possíveis limitações do estudo, quais devem ser consideradas tangem especialmente ao tipo de estudo, uma vez que é de caráter retrospectivo assim apresenta um controle limitado levando em consideração o tamanho da amostra e analise de variáveis.

Ademais conforme dados descritos no presente trabalho pode- se concluir, especialmente analisando o dado em que o estadiamento foi descoberto em estágios já avançados nos pacientes analisados, que o presente trabalho comprova a necessidade de aprimoramento de diagnóstico precoce e rastreamento de pacientes com fatores de riscos importantes para o desenvolvimento da doença.

\section{Referências}

[1] Corte Z, Casado MM, Augé JM, Filella X, Escudero JM, Molina V, Molina R. Marcadores tumorales en neoplasias gástricas. 2010;1756(1):45-52. 
[2] Inca. Incidência de Câncer no Brasil Estimativa 2018. Rio de Janeiro: Ministério da Saúde; 2018.

[3] Ikeda Y, Mori M, Kamakura T, Haraguchi Y, Saku M, Sugimachi K. Improvements in diagnosis have changed the incidence of histological types in advanced gastric cancer. British Journal of Cancer. 1995;72(2):424-6. doi: https:/ / doi.org/10.1038/bjc.1995.349.

[4] Zhu AL, Sonnenberg A. Is Gastric Cancer Again Rising? Journal of clinical gastroenterology. 2012;46(9):804-6. doi: 10.1097/MCG.0b013e3182604254.

[5] Roesch-Dietlen F, Jiménez-García VA, Remes-Troche JM, Rubio-Arce JF, López-Salinas A, Ruiz-Juárez I, GrubePagola P, Silva-Cañetas CF. Comportamiento epidemiológico de las neoplasias malignas del tracto digestivo en un periodo de 5 años en Veracruz, México. Revista de Gastroenterología de México. 2012;77(1):1-50.

[6] Lever-Rosas CD, Silva-Ortiz J, Almanza-Muñoz JJ. Cáncer gástrico. Abordaje multidisciplinario, cirugía, psico-oncología y calidad de vida. Revista de Sanidad Militar. 2014;68(3):177-88.

[7]Hamada GS, Kowalski LP, Nishimoto IN et al. Risk factors for stomach cancer in Brazil (II): a case-control study among Japanese Brazilians in São Paulo. Japanese Journal of Clinical Oncology. 2002;32(8):284-90. doi: https://doi.org/10.1093/jjco/hyf061.

[8]Bray F, Ferlay J, Soerjomataram I, Siegel RL, Torre LA, Jemal A. Global cancer statistics 2018: GLOBOCAN estimates of incidence and mortality worldwide for 36 cancers in 185 countries. CA Cancer J Clin. 2018 Nov;68(6):394-424. 10.3322/caac. 21492.

[9]Arregi MMU, Férrer DPC, Assis ECV de, Paiva FDS de, Sobral LBG, André NF, Silva TC da. Perfil clinico epidemiológico das neoplasias de estomago atendidas no Hospital do Câncer do Instituto do Câncer do Ceará, no período de 2000-2004. Revista Brasileira de Cancerologia. 2009; 55(2):121-8. doi:

https:/ / doi.org/10.32635/2176-

9745.RBC.2009v55n2.1641

[10]Diniz LL, Santos SLF, Arraes MLB de M, Barros KBNT. Prevalência de doenças gástricas não infecciosas em idosos. Mostra Científica da Farmácia. 2017;3(1).

[11]Guimarães AVS, Sales ML. Fatores de risco no desenvolvimento de câncer gástrico. Revista Brasileira de Ciências Da Vida. 2017;59(1):1-24.

[12] Brasil. IBGE. Pesquisa Nacional de Saúde 2013: acesso e utilização dos serviços de saúde, acidentes e violências: Brasil, grandes regiões e unidades da federação. Rio de Janeiro: IBGE; 2015. 100 p.

[13] Crew KD, Neugut AI. Epidemiology of gastric cancer. World Journal of Gastroenterology. 2006;12(3):354-62. doi: 10.3748/wjg.v12.i3.354

[14]Basaran H, Koca T, Cerkesli AK, Arslan D, Karaca S. Treatment outcomes and survival study of gastric cancer patients: a retrospective analysis in an 
endemic region. Asian Pac J Cancer Prev. 2015;16(5):2055-60. doi: 10.7314/apjcp.2015.16.5.2055.

[15] Nomura, A. Stomach cancer. In: Schottenfeld, D.; Fraumeni, J. F. Cancer Epidemiology and Prevention. New York: Oxford University Press; 1996. p. 707-724.

[16] Wang XQ, Terry PD, Yan H. Review of salt consumption and stomach cancer risk: Epidemiological and biological evidence. World Journal of Gastroenterology. 2009;15(18):2204-13. doi: 10.3748/wjg.15.2204

[17] González C. Cáncer gástrico: factores de riesgo, carcinogénesis, bases moleculares. Gen. 2010;64(3):214-20.

[18] Csendes A, Figueroa M. Situación del cáncer gástrico en el mundo y en Chile. Revista Chilena de Cirugía. 2017;69(6):502-7. doi: http://dx.doi.org/10.1016/j.rchic.2016.1 0.014

[19] Brasil. Ministério da Saúde. Secretaria de Vigilância em Saúde. Departamento de Análise em Saúde e Vigilância de Doenças não Transmissíveis. Vigitel: Brasil 2018. Brasília: Ministério da Saúde; 2019; 132 p.

[20] Laranjeira R, Madruga CS, Pinsky I, Caetano R, Mitsuhiro SS. II Levantamento Nacional de Álcool e Drogas - LENAD 2012. São Paulo: Instituto Nacional de Ciência e Tecnologia para Políticas Públicas de Álcool e Outras Drogas (INPAD); UNIFESP; 2014.
[21]Barstad B, Sørensen TI, Tjønneland A, Johansen D, Becker U, Andersen IB, Grønbaek M. Intake of wine, beer and spirits and risk of gastric cancer. Eur J Cancer Prev. 2005 Jun;14(3):239-43. doi: 10.1097/00008469-200506000-00007.

[22] Tramacere I, Negri E, Pelucchi C, Bagnardi V, Rota M, Scotti L, Islami F, Corrao G, La Vecchia C, Boffetta P. A meta-analysis on alcohol drinking and gastric cancer risk. Ann Oncol. 2012 Jan;23(1):28-36.

doi: 10.1093/annonc/mdr135.

[23] Galbiatti ALS, Padovani-Junior JA, Maníglia JV, Rodrigues CDS, Pavarino EC, Gloni-Bertollo EM. Câncer de cabeça e pescoço: causas, prevenção e tratamento. Brazilian Journal of Otorhinolaryngology. 2013;79:239-47. doi: https://doi.org/10.5935/18088694.20130041

[24]Brenner $H$, Arndt $V$, Stürmer $T$, Stegmaier C, Ziegler H, Dhom G. Individual and joint contribution of family history and Helicobacter pylori infection to the risk of gastric carcinoma. Cancer. 2000 Jan 15;88(2):274-9. doi: 10.1002/(sici)10970142(20000115)88:2<27 4::aid-cncr5>3.0.co;2-9.

[25]Fock KM. Review article: the epidemiology and prevention of gastric cancer. Alimentary Pharmacology \& Therapeutics. 2014;40(3):250-60. doi: https://doi.org/10.1111/apt.12814.

Conflito de interesse: Os autores declaram não haver conflitos de interesse.

Agradecimentos: Agradecemos a toda equipe pelo excelente manejo multidisciplinar e especialmente ao 
Hospital de Clínicas de Passo Fundo na contribuição e fornecimento de dados para realização do presente trabalho.

Financiamento: Não se aplica.

Como citar este artigo: Pastorello J, Lando E, Messaggi ALM, Bueno MR, Cechetti CP, Amaral CP, Cogo DC, Rosa Filho LA. Perfil clínico-epidemiológico de pacientes diagnosticados com câncer gástrico atendidos na especialidade de oncologia do Hospital de Clínicas de Passo Fundo - RS. Brazilian Journal of Case Reports. 2021 Jul-Sep; 01(3):27-37. 